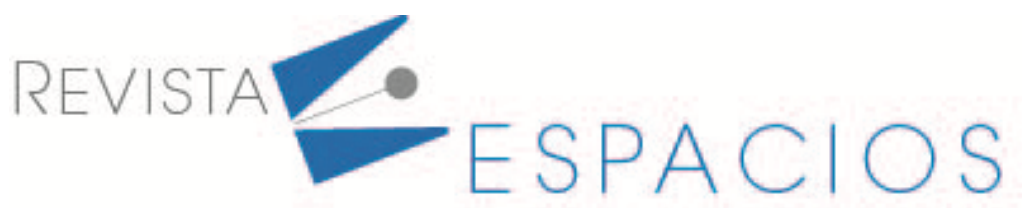

\title{
Análisis del plan de marketing en empresas de la agroindustria alimentaria en Colombia
}

\section{Analysis of the marketing plan in food agroindustry companies in Colombia}

\author{
ORTIZ, Laura T. ${ }^{1}$ \\ MELO, Ligia I. ${ }^{2}$ \\ NOVA, Luis C. ${ }^{3}$
}

\begin{abstract}
Resumen
El propósito de la investigación fue analizar el nivel de utilización del Plan de Marketing en agroindustrias de la guayaba en Boyacá y Santander, Colombia. Se realizó un análisis descriptivo de información de tipo cuantitativa y mediante la aplicación de encuestas estructuradas a empresarios del sector, se pudo comprender cómo los elementos básicos de dicho plan pueden contribuir al logro de los objetivos organizacionales y del mismo modo fue posible identificar qué partes deben ser mejoradas en el futuro. Palabras clave: plan de marketing, agroindustria, marketing estratégico, marketing operativo.
\end{abstract}

\begin{abstract}
The purpose of the investigation was to analyze the level of utilization of the Marketing Plan in guava agroindustries in Boyacá and Santander, Colombia. A descriptive analysis of quantitative information was carried out and through the application of structured surveys to businessmen in the sector, it was possible to understand how the basic elements of said plan can contribute to the achievement of organizational objectives and in the same way it was possible to identify which parts they must be improved in the future.
\end{abstract}

Key words: marketing plan, agroindustry, strategic marketing, operational marketing.

\section{Introducción}

La agroindustria en Colombia tuvo sus inicios en el año 1904, gracias a la tecnología y el comercio, para el año de 1945 la industria de alimentos y bebidas representaba el 47\% del valor de la producción del sector industrial y generaba un $27.2 \%$ de empleo en el país; según informes del Centro de estudios ganaderos y agrícolas, en la década de 1985 a 1996 alcanzó el mayor crecimiento; en la actualidad se ha avanzado en la consolidación del sector, mostrando que la importancia de la agroindustria radica en la generación de empleo, ingresos y aportes al PIB, además de facilitar el enlace entre los sectores agropecuario y de servicios; motivos por los cuales se

\footnotetext{
${ }^{1}$ Investigadora Grupo Cide, Universidad Pedagógica y Tecnológica de Colombia. lauratatiana.ortiz@uptc.edu.co / luuortt@hotmail.com. https://orcid.org/0000-0003-2269-3736

2 Profesora Asociada. Escuela de Administración de Empresas, Universidad Pedagógica y Tecnológica de Colombia Sede Seccional Sogamoso. Investigadora grupo Cide. ligia.melo@uptc.edu.co. https://orcid.org/0000-0002-4994-776X.

3 Profesor Asistente. Escuela de Administración de Empresas, Universidad Pedagógica y Tecnológica de Colombia Sede Seccional Sogamoso. Investigador grupo Gestor. Iuis.nova01@uptc.edu.co. https://orcid.org/0000-0002-0978-1320.
} 
considera un tema de interés general, tanto que los gobiernos lo incluyen en sus planes de desarrollo (González, 2015).

El presente artículo es el resultado de un proyecto de investigación del grupo Competitividad innovación y desarrollo empresarial (Cide), adscrito a la Universidad Pedagógica y Tecnológica de Colombia, cuyo objetivo fue analizar el plan de marketing en empresas de la agroindustria alimentaria, centrado en la agroindustria de la guayaba en los municipios de Vélez y Barbosa en el departamento de Santander y Moniquirá en el departamento de Boyacá, donde su principal producto es el bocadillo del cual su historia data del siglo XVI (Gobernación de Santander, 2017).

La agroindustria de la guayaba ha convocado a diferentes entes e instituciones a apoyar y aportar en su mejoramiento, como el Servicio Nacional de Aprendizaje (SENA), Cadena Productiva de la guayaba y la Agencia de Desarrollo Económico Local (Adel) Vélez, entre otras; logrando un tejido social fuerte evidenciado en la creación de asociaciones y beneficios significativos como la denominación de origen para el bocadillo veleño (Superintendencia de Industria y Comercio, 2017), así como el desarrollo de proyectos estratégicos para mejorar toda la cadena productiva y expandir sus mercados, dado que sus productos llegan a diferentes partes del país y se exportan a Europa, Estados Unidos y América Central (Ministerio de Agricultura y Desarrollo Rural, 2018; Adel Vélez, 2015).

En Colombia, la guayaba ocupa el cuarto lugar en consumo en fresco con un 14\% (Alonso, Ordoñez y Rivera, 2017), con perspectiva de aumento debido a su inclusión como alimento priorizado en el plan nacional de seguridad alimentaria y nutrición 2012- 2019, en tanto que el mercado de los productos procesados es superior comparado con el producto fresco, siendo esta industria una de las más dinámicas del mundo; pues su producción mundial en 2011 fue de 520.737 millones de toneladas, por un valor equivalente a USD 4.443 millones; con una proyección de crecimiento anual del 8.1\% para el periodo comprendido del 2011 al 2020 (Ministerio de Agricultura y Desarrollo Rural, 2015).

Los países productores de guayaba son Egipto, Brasil, Colombia, Perú, Ecuador, India, Sudáfrica, Estados Unidos, México, Filipinas, Venezuela, Costa Rica, Cuba y Puerto Rico; en Colombia los departamentos con mayores producciones de guayaba común son Santander, Atlántico, Boyacá, Valle, Córdoba y Tolima, y en guayaba pera, Meta, Santander, Valle, Caldas y Cauca (Ministerio de Agricultura y Desarrollo Rural, 2018), cuyo consolidado de áreas sembradas y producción del 2014 a 2018 se muestra en la tabla 1.

Tabla 1

Áreas cultivadas y producción de guayaba en Colombia

\begin{tabular}{ccccccc}
\hline & \multicolumn{2}{c}{ Guayaba Común } & \multicolumn{2}{c}{ Guayaba Pera } & \multicolumn{2}{c}{ Producción Total } \\
\cline { 2 - 7 } Año & $\begin{array}{c}\text { Área Sembrada } \\
(\mathrm{Ha})\end{array}$ & $\begin{array}{c}\text { Producción } \\
(\mathrm{T})\end{array}$ & $\begin{array}{c}\text { Área Sembrada } \\
(\mathrm{Ha})\end{array}$ & $\begin{array}{c}\text { Producción } \\
(\mathrm{T})\end{array}$ & $\begin{array}{c}\text { Área Sembrada } \\
(\mathrm{Ha})\end{array}$ & $\begin{array}{c}\text { Producción } \\
(\mathrm{T})\end{array}$ \\
\hline 2014 & 10.746 & 73.783 & 6.562 & 63.226 & 17.308 & 137.009 \\
2015 & 10.320 & 67.707 & 6.847 & 66.488 & 17.167 & 134.195 \\
2016 & 8.647 & 70.054 & 7.262 & 48.162 & 15.909 & 118.216 \\
2017 & 9.882 & 92.425 & 7.403 & 55.493 & 17.285 & 147.918 \\
2018 & 10.475 & 97.970 & 7.847 & 58.822 & 18.322 & 156.792 \\
\hline
\end{tabular}

Fuente: Ministerio de Agricultura y Desarrollo Rural. Cifras sectoriales 2018

Teniendo en cuenta lo expuesto anteriormente y reconociendo la importancia de la agroindustria alimentaria, así como del plan de marketing para la sostenibilidad y competitividad de las empresas, se desarrolló la presente investigación. 


\subsection{Referentes teóricos}

El Plan de Marketing surge como uno de los principales activos de la organización en la obtención de resultados, bajo una visión actual y de futuro que sirve para precisar sus directrices con máximas garantías y menor grado de incertidumbre (Muñiz González, 2015; Artega, Ruiz, y Navas, 2017). Acevedo, Cotes, Giraldo, Juliao, Martínez, Ortíz, y Silva (2016) recalcan en la existencia de una estructura guía, mas no un modelo genérico para la elaboración del plan de marketing, dado que cada plan debe construirse con base en los recursos, capacidades, requerimientos empresariales, además de las condiciones del mercado, por tanto, permite flexibilidad (Artega, Ruiz, y Navas, 2017), como se muestra en la figura 1, esquema que facilita su adaptación a las necesidades de cada organización.

Figura 1

Componentes del plan de marketing

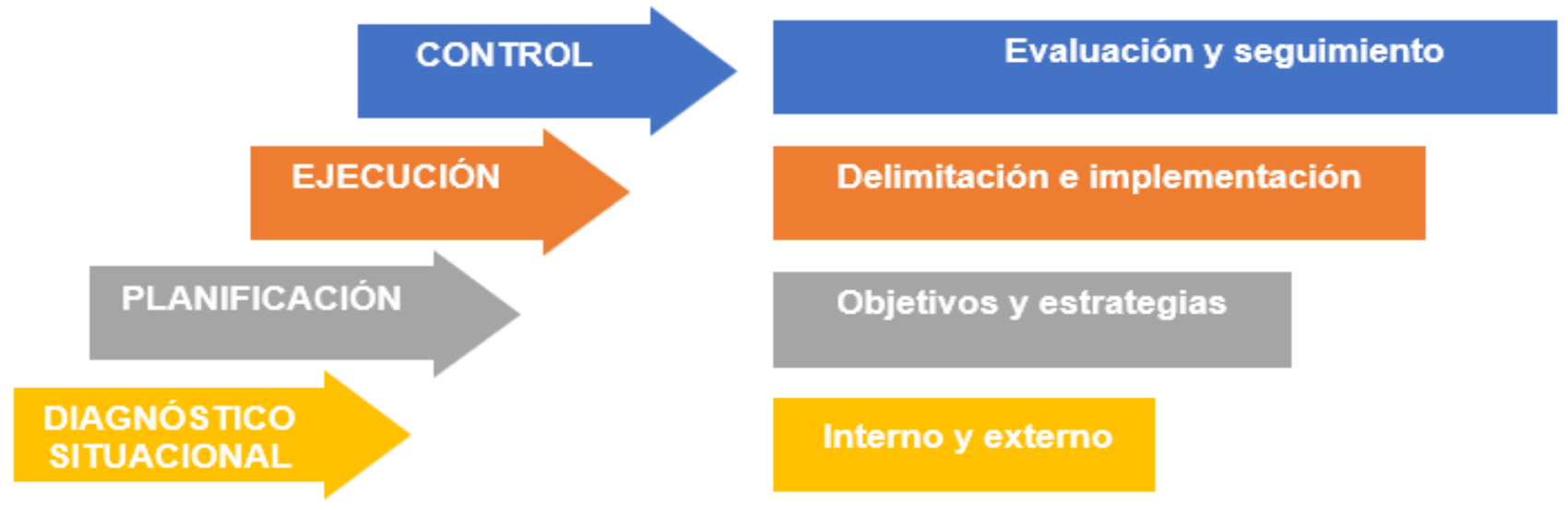

Fuente. Elaboración propia

El plan de marketing con el apoyo de la organización y de los recursos existentes (Kotler y Lane, 2016) busca establecer un diálogo entre empresa y cliente, para generar valor hacia el cliente en materia de satisfacción y cubrimiento de necesidades, partiendo desde los fundamentos tradicionales hasta las nuevas tendencias (Viteri, Herrera, y Bazurto, 2017) para lo cual, es preciso reconocer y enfatizar en las dos dimensiones del plan de marketing (figura 2), la estratégica soportada en los valores de la compañía trabaja para saber dónde está, a dónde quiere ir y de qué manera lo hará, desde el análisis y la reflexión del marketing brinda soluciones a largo plazo; para satisfacer las necesidades de los stakeholders, así como la búsqueda de estrategias orientadas a la exploración permanente de oportunidades en el mercado (Estrada, Elidea, Cifuentes, y Ayovi, 2017; Toca Torres, 2009).

La segunda dimensión conocida como operativa, en la cual se ejecutan las estrategias precisas para alcanzar los objetivos propuestos (Ferrell y Hartline, 2018; Kotler y Lane, 2016; Monferrer Tirado, 2013), está relacionada con llevar a la acción las operaciones de venta, distribución, comunicación y la verificación de que lo planeado se ejecute. Desde esta perspectiva se persigue el objetivo de incrementar en el corto plazo la cuota de mercado, cuyo logro tendrá un efecto directo en las ventas, los ingresos y las utilidades de la organización; por tal razón, se constituye en el aspecto visible del marketing y se apoya fundamentalmente en medios tácticos (Toca Torres, 2009); donde la satisfacción del cliente ya sea a través del servicio y la calidad, son fuentes de valor para la empresa con clientes cada vez más exigentes (Zárraga, Molina, y Corona, 2018). 
Figura 2

Dimensiones estratégica y operativa del plan de marketing
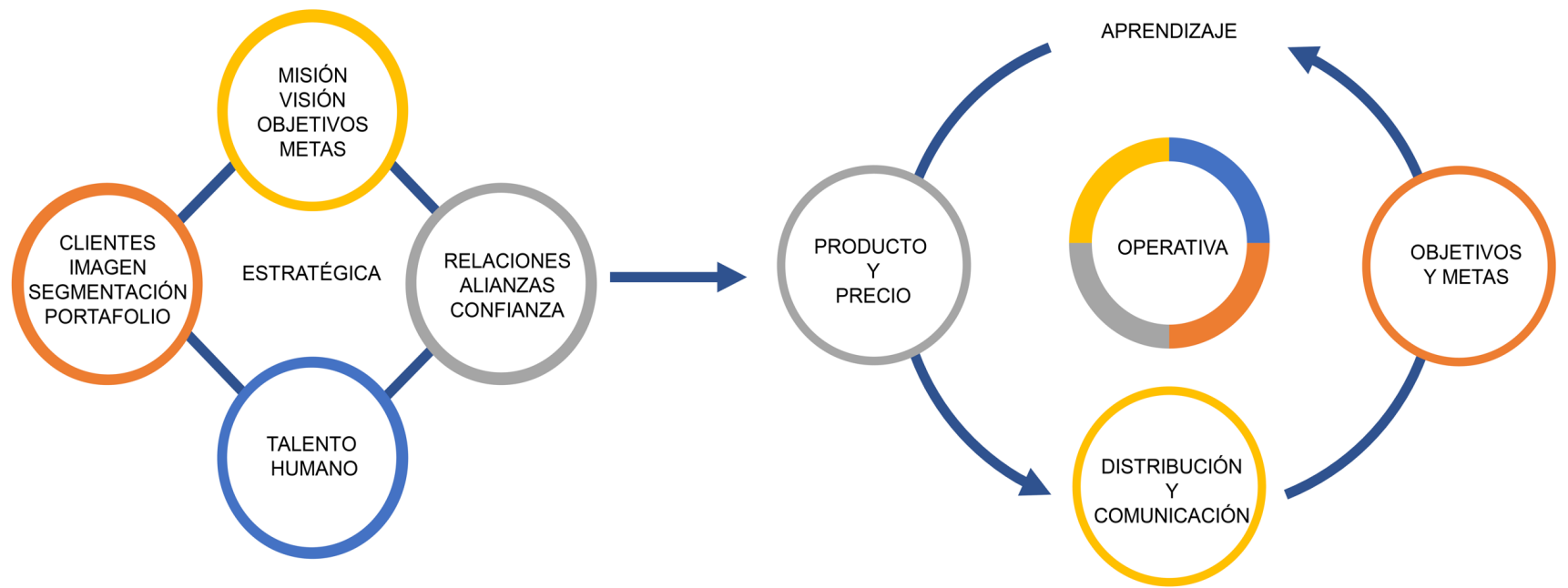

Fuente. Elaboración propia

La elaboración de un plan de marketing inicia con el diagnóstico situacional, también conocido como análisis estratégico, que según Serna (2014) y Thompson, Strickland, Janes, Sutton, Peteraf, y Gamble (2018), es un proceso crucial para la perspectiva de futuro partiendo de conocer la posición actual de la compañía; este proceso sistémico y analítico permite conocer la situación actual de una organización en sus entornos tanto interno como externo, dejando ver las áreas precisas para realizar cambios camino al mejoramiento continuo (Maldonado Pinto, 2013) y tiene como función guiar a la empresa en el proceso de adopción y formulación de políticas y estrategias efectivas que le han de permitir conseguir sus objetivos y consolidación de su futuro (Munera y Rodríguez, 2020; Borges Andrade, Escobar, Palomino, Saldaña, y De Souza Silva, 1995).

El diagnóstico interno comprende la evaluación de recursos y capacidades de la organización, empieza por el direccionamiento estratégico en relación a la misión, visión y objetivos (Serna, 2014; Thompson et al., 2018); le sigue el análisis profundo del mercado y una rigurosa reflexión estratégica (Munera y Rodríguez, 2020) sobre los aspectos propios del mercado que se encargan de dar o no un ambiente de estabilidad; como son clientes, investigaciones de mercados, segmentación del mercado (Zárraga Cano, Molina, y Corona, 2013), así como el relacionamiento con stakeholders (García y Pérez, 2018; Taipe y Pazmiño, 2015; Lambin, Gallucci y Sicurello, 2009) dado por el concepto de ecosistema de mercado; de igual forma el enfoque de marketing interno o gestión del talento humano dentro de la organización (Sainz de Vicuña, 2018; Jiménez y Gamboa, 2016).

Cada organización de acuerdo con su filosofía y cultura cuenta con métodos específicos para atraer, retener, motivar, desarrollar y fidelizar el talento humano; evidenciados en los distintos procesos y políticas de selección e incorporación de nuevos trabajadores, diseño y manejo de la estructura de los puestos de trabajo, capacitación de la fuerza de trabajo, esquema y estructura de remuneración, beneficios e incentivos, medición de desempeño de un colaborador en un determinado periodo, e identificación de percepción y motivación de los trabajadores (Acevedo Navas, et al., 2016; Jiménez y Gamboa, 2016; Stanton, Etzel, y Walker, 2007).

Uno de los resultados del diagnóstico estratégico interno es un consolidado de fortalezas y debilidades; las primeras consideradas como características intrínsecas de los insumos, procesos y productos que apoyan a la organización para aprovechar las oportunidades o les permite protegerse de las amenazas provenientes del contexto, mientras que las segundas, son aquellas particularidades de insumos, procesos y productos que no 
apoyan a la organización para aprovechar las oportunidades y protegerse de las amenazas provenientes del contexto (Borges et al., 1995).

Por su parte, el diagnóstico externo permite determinar e identificar los factores y elementos del entorno que afectan e influyen en las decisiones de la organización, ya sean políticos, económicos, sociales, tecnológicos o ambientales, legales y culturales; reconociendo que todos estos aspectos se encuentran fuera del control de la organización (Zenea, Arcia, y Guerra, 2019; Barrionuevo, Cárdenas, Cárdenas, Cabezas, y Sandoval, 2019), como resultado de este análisis es posible identificar oportunidades y amenazas para la consecución de los objetivos de la organización.

Por otro lado la dimensión operativa se concentra en el desarrollo y materialización de las acciones específicas y puesta en marcha de las estrategias propuestas, convirtiéndolas en acciones y decisiones tácticas y políticas comerciales que contemplen las políticas de producto, precio, distribución y comunicación en pro del objetivo de la empresa, lo cual implica medición de resultados, verificación del grado de cumplimiento de objetivos y metas, y adopción de oportunas medidas correctivas (Acevedo Navas et al., 2016; Munera y Rodríguez, 2020; Stanton, Etzel, y Walker, 2007).

El segundo paso en la elaboración del plan de marketing es la planificación, que comprende la fijación de objetivos y metas, así como la elección de la estrategia, aunque algunos autores lo muestran en pasos separados (Monferrer Tirado, 2013; Sainz de Vicuña, 2018) sin embargo, al plantear los objetivos con la aplicación de sus características es lógico e imprescindible pensar en las estrategias necesarias para lograrlos, por lo cual, es más afín su cercanía y aquí se presenta de manera conjunta.

Una vez realizado el diagnóstico estratégico su resultado permite conocer el real estado del negocio, así como mostrar que tanto se deben replantear los objetivos y metas o plantear nuevos, y en este orden de ideas las estrategias; donde los objetivos y metas se deben formular de manera que se cristalicen dentro de lo programado (Monferrer Tirado, 2013) y las estrategias faciliten establecer el lugar esperado en el mercado y todo lo que realmente se necesita para lograrlo (Acevedo Navas et al., 2016). Dos herramientas que facilitan este paso es la matriz de fortalezas, oportunidades, debilidades y amenazas- FODA (Estrada et al, 2017; Zenea, Arcia, y Guerra, 2019;) complementada con el análisis CAME (corregir, afrontar, mantener y explotar) herramientas que permiten confrontar los resultados del análisis interno con los del externo y formular las estrategias con miras a alcanzar los objetivos y metas propuestas (Sánchez, 2015).

Luego de la definición de objetivos, metas y las estrategias, viene la etapa de ejecución que es el tercer paso en el esquema del plan de marketing propuesto, entonces, es preciso realizar la delimitación y definición de los programas para llevar a cabo la estrategia elegida, incluyendo actividades concretas, su planificación temporal, presupuesto y asignación de responsabilidades para, producto, precio, distribución y comunicación; lo cual implica poner en funcionamiento la estrategia a través del diseño de acciones que ayuden a alcanzar los objetivos fijados (Monferrer Tirado, 2013), etapa que precisa de la coordinación de la estructura organizativa, talento humano, procesos de dirección y cultura empresarial (Munera y Rodríguez, 2020), enfatizando que es a través de una perfecta ejecución de la estrategia que se logra reconocimiento por parte del consumidor, preferencia, compra y como consecuencia su lealtad (Villegas y Escalante, 2016).

A la ejecución le sigue la etapa de control, donde se busca asegurar que lo planeado se cumpla, que los resultados esperados se alcancen y que los procesos se desarrollen tal cual fueron planeados; el control indica cómo se va a medir la evolución de los objetivos, por tanto, conlleva a realizar un seguimiento a través de indicadores y comparación de los resultados; el análisis de las causas de estos y la toma de medidas correctivas para asegurar el cumplimiento de lo proyectado (Monferrer Tirado, 2013). 
Todo este proceso, desde el diagnóstico situacional, la planificación, ejecución y control, demandan capacidad de gestión, habilidades técnicas, así como un amplio sentido de innovación, sensibilidad y coordinación para alcanzar los objetivos y metas propuestos (Acevedo Navas et al., 2016; Lambin, Gallucci, y Sicurello, 2009).

\section{Metodología}

La presente investigación se desarrolló bajo una perspectiva cuantitativa y método descriptivo cuyo objetivo fue analizar el plan de marketing en las empresas de agroindustria de la guayaba; para la recolección de la información se diseñó un formulario de encuesta, estructurado en dos partes, la primera recoge la información correspondiente a la clasificación general de las empresas y sus gerentes, la segunda parte en concordancia con lo expuesto en el marco teórico y las agrupaciones a analizar tanto del marketing estratégico, como operativo, enfatizando en el análisis interno (tabla 2).

Tabla 2

Estructura de encuesta aplicada en empresas de la agroindustria de guayaba en Colombia

\begin{tabular}{|c|c|c|c|}
\hline & Variables & Aspectos por analizar & $\begin{array}{c}\text { No. de } \\
\text { preguntas }\end{array}$ \\
\hline \multirow{4}{*}{ 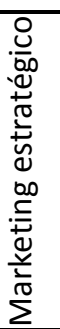 } & Direccionamiento & Misión, visión, imagen, objetivos, metas & 5 \\
\hline & Mercados & $\begin{array}{l}\text { clientes, investigación, segmentación, portafolio, } \\
\text { tabla nutricional }\end{array}$ & 8 \\
\hline & Relacionamiento & Alianzas, proveedores, agremiación, competidores & 6 \\
\hline & Talento humano & $\begin{array}{l}\text { Selección, remuneración, formación, valoración } \\
\text { desempeño, estructura y puestos de trabajo }\end{array}$ & 6 \\
\hline \multirow{3}{*}{ 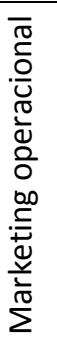 } & Producto y precio & $\begin{array}{l}\text { Calidad, precio, registro Invima, } \\
\text { planifica y presupuesta el mercadeo }\end{array}$ & 6 \\
\hline & $\begin{array}{l}\text { Distribución y } \\
\text { comunicación }\end{array}$ & $\begin{array}{l}\text { Canales de distribución, línea telefónica, directorio, } \\
\text { página Web, Facebook, Instagram }\end{array}$ & 9 \\
\hline & Verificación y control & $\begin{array}{l}\text { Beneficios de planificación, objetivos y } \\
\text { metas verificables, utilidades }\end{array}$ & 4 \\
\hline
\end{tabular}

La investigación se llevó a cabo en los municipios de Vélez y Barbosa pertenecientes al departamento de Santander y Moniquirá en el departamento de Boyacá, dado que allí es donde se ubica el mayor número de empresas de agroindustria de la guayaba. Según el Ministerio de Agricultura y Desarrollo Rural (2018), existen 131 fábricas, en las cuales se estableció contacto con los gerentes y se hizo la invitación a hacer parte de la investigación, se obtuvo respuesta positiva de 47 y fue sobre las cuales se trabajó; en el 2019 a los gerentes generales se le aplicó la encuesta acorde a la estructura mostrada en la tabla 2.

Una vez obtenida la información se procedió a la codificación y tabulación mediante el paquete estadístico SPSS (Statistical Package for the social Sciences) y Excel, herramientas que facilitan el análisis y presentación de resultados en tablas y figuras acorde a los requerimientos de la investigación.

\section{Resultados}

El análisis se centró en la parte interna de las organizaciones; en primer lugar, se muestran resultados de los aspectos generales de las empresas y sus gerentes, posteriormente lo correspondiente al marketing estratégico 
y operativo, y luego se presenta un resumen de fortalezas y debilidades, complementado con algunos factores de la parte externa en cuanto a oportunidades y amenazas.

De las empresas de la agroindustria de la guayaba, el $51,1 \%$ está dirigida por mujeres y el 48,9\% por hombres, donde el $30 \%$ cuenta con un título profesional universitario y el $87,2 \%$ son empresas familiares; en cuanto al tamaño acorde al número de empleados el $100 \%$ se ubica en la categoría de Mipymes, el $53,3 \%$ son microempresas, el 42,6\% pequeñas y el 4,3\% medianas empresas; en ubicación por municipios el 48,9\% en Vélez, 36,2\% en Moniquirá y el 14,9\% en Barbosa; frente al cumplimiento de requisitos de formalización el $97,9 \%$ cuenta con Registro Único Tributario (RUT) ante la Dirección de Impuestos y Aduanas Nacionales (Dian); el 83\% tiene registro mercantil adscrito en las respectivas Cámaras de Comercio.

Para categorizar la antigüedad de estas empresas (tabla 3) se establecieron rangos con intervalos de 5 hasta 30 años, luego de a 10 años y finalmente una categoría de 51 a 79 años que corresponde a las de mayor antigüedad con un 17\%, en igual porcentaje al rango de 21 a 25 años, información significativa que muestra que el 100\% ya superó los cinco años de vida, dado que la mayor mortalidad de empresas se presenta en esos primeros años.

Tabla 3

Antigüedad de las empresas de agroindustria de la guayaba en Colombia

\begin{tabular}{|c|c|c|c|c|c|}
\hline Rango en años & Frecuencia & Porcentaje & Rango en años & Frecuencia & Porcentaje \\
\hline 6 a 10 & 4 & 8,5 & 26 a 30 & 4 & 8,5 \\
\hline 11 a 15 & 5 & 10,6 & 31 a 40 & 6 & 12,8 \\
\hline 16 a 20 & 6 & 12,8 & 41 a 50 & 6 & 12,8 \\
\hline 21 a 25 & 8 & 17 & 51 a 79 & 8 & 17 \\
\hline
\end{tabular}

Fuente: Elaboración propia

\subsection{Marketing estratégico}

La primera agrupación analizada es el direccionamiento estratégico (tabla 4), allí se observa que el cumplimiento en cuanto a misión y visión está por encima del 95\%, en imagen y objetivos superior al $85 \%$, resultados que dejan ver que las empresas tienen claridad sobre su razón de ser, así como a dónde se quieren proyectar, resaltando la importancia de formular finalidades y propósitos claros que apunten a la perdurabilidad y sostenibilidad de las empresas como lo precisan Serna (2014); Thompson et al (2018), desde luego alineadas con las estrategias y su puesta en marcha, dado que es un proceso integral.

Tabla 4

Cumplimiento del direccionamiento estratégico en empresas de agroindustria de la guayaba en Colombia

\begin{tabular}{lcc}
\hline \multicolumn{1}{c}{ Aspectos valorados } & Frecuencia & Porcentaje \\
\hline Tiene misión definida & 46 & 97,9 \\
Cuenta con visión & 45 & 95,7 \\
Buena Imagen de la empresa & 41 & 87,2 \\
Tiene objetivos & 40 & 85,1 \\
Tiene metas establecidas & 31 & 66,0 \\
\hline
\end{tabular}

Fuente: Elaboración propia 
Continuando con el análisis, se encuentran los aspectos del mercado (figura 3), en conocimiento de clientes y análisis de sus necesidades su cumplimiento está en el 98\%, condición favorable para las empresas, pues conocer, entender y responder a sus expectativas son aspectos claves en el éxito de una compañía (Munera y Rodríguez, 2020); le sigue investigación de mercados con el $85 \%$, que a su vez es parte fundamental para el conocimiento de clientes actuales y potenciales, además de contribuir al posicionamiento de la marca. Los resultados correspondientes a segmentación, importancia dada a las habilidades en mercadotecnia y contar con tabla nutricional, su desempeño se ubicó entre el 66 y 72,3\%; en tanto que el 53,2\% de las empresas cuenta con un departamento de mercadeo, resultado dado por el tamaño de las empresas a sabiendas que el $53 \%$ son microempresas, y una debilidad notoria si es que solo el $42,6 \%$ cuenta con portafolio de productos.

Figura 3

Cumplimiento de los aspectos de mercados en empresas de agroindustria de la guayaba en Colombia

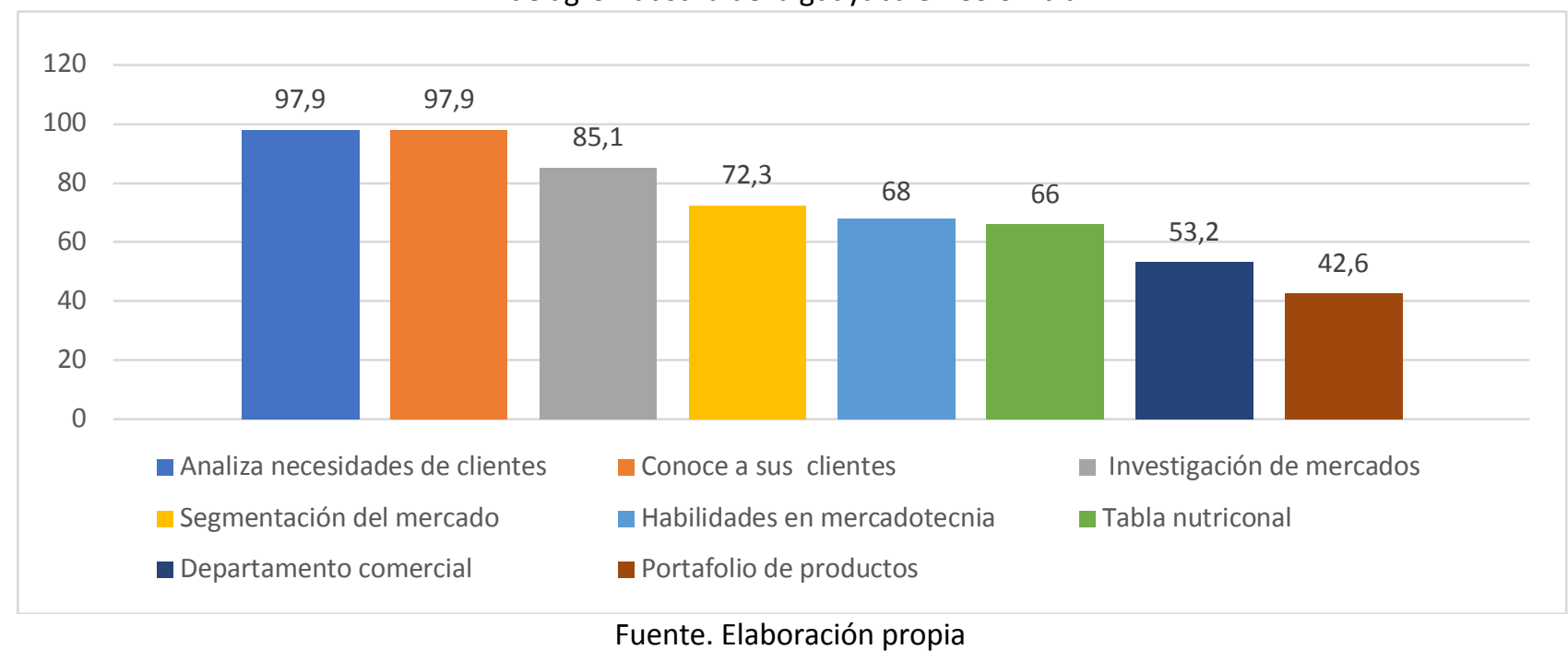

El relacionamiento y retomando el concepto de ecosistema de mercado de Lambin, Gallucci, y Sicurello (2009) donde se tienen en cuenta las relaciones con diferentes stakeholders; los resultados de esta agrupación (figura 4) muestran que el mejor porcentaje lo obtuvo la confianza en proveedores con el 93,6\%, seguida de la identificación de competidores con el 89,4\% y contar con buena posición frente a la competencia con un 87,2\%; en tanto que pertenecer a una asociación y conocimiento de estrategias de competidores está presente en el $55,3 \%$ y $53,2 \%$ respectivamente; y tan solo en el $31,9 \%$ se cuenta con alianzas, situación que se interpreta como debilidad dado el tamaño de las empresas, pues estas alianzas pueden ayudar a incrementar las ventas, disminuir costos en algunos aspectos y desde luego obtener resultados positivos, bien sea por compartir almacenes, compra de insumos, transporte, entre otros, y fortalecer las relaciones gana-gana, como lo mencionan García y Pérez (2018). 
Figura 4

Cumplimiento en aspectos de relacionamiento en empresas de agroindustria de la guayaba en Colombia

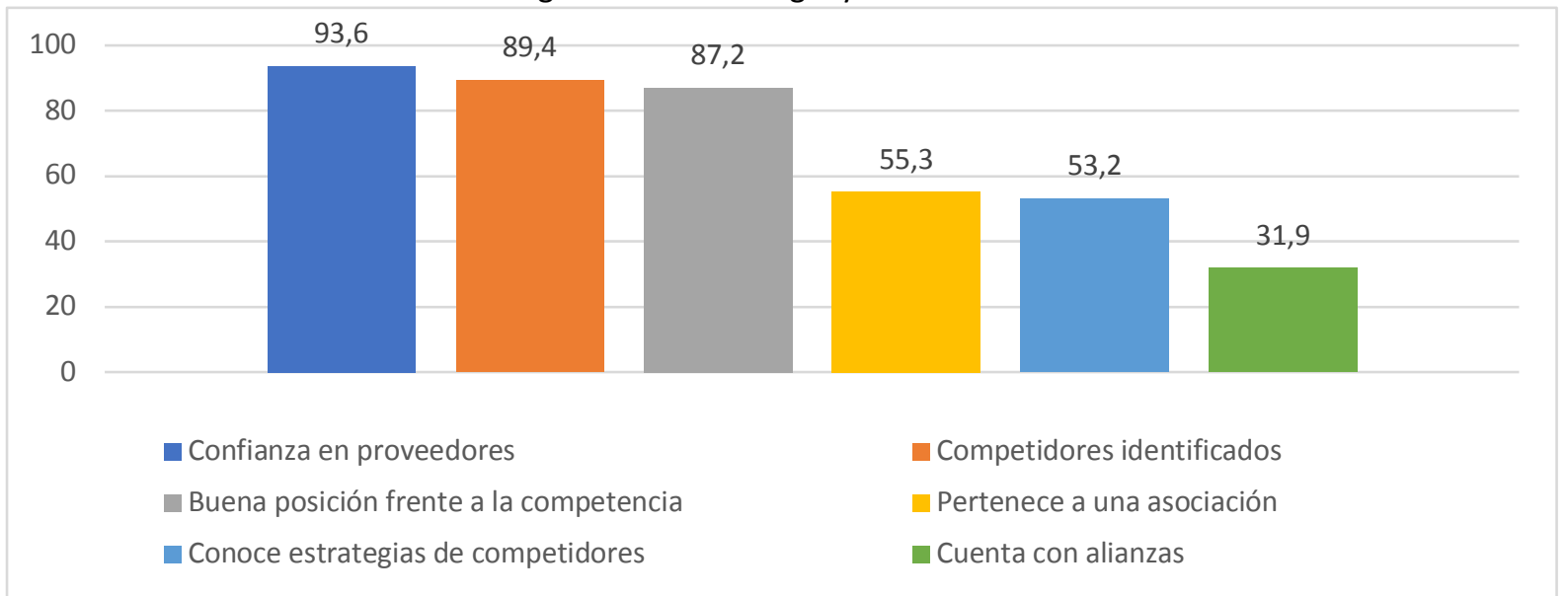

Fuente. Elaboración propia

En talento humano o cliente interno los resultados (figura 5), como lo advierten Sainz de Vicuña (2018); Jiménez y Gamboa (2016), el cliente interno es fundamental para el cumplimiento de los objetivos y metas de mercado, donde cada empresa desde su filosofía y cultura establece métodos específicos para atraer, retener, motivar, desarrollar y fidelizar el talento humano, en este caso el cumplimiento de los aspectos analizados su porcentaje de cumplimiento va del 93,6\%, correspondiente a la remuneración salarial acorde al desempeño, hasta 51,1\% en formación, capacitación del personal y valoración cualitativa del desempeño; de igual forma se observa la presencia de aspectos importantes como programas de salud ocupacional y la selección de personal, que aunque un alto número son microempresas, los aspectos analizados en este ítem se cumplen en más del $50 \%$ de las empresas.

Figura 5

Cumplimiento en aspectos de talento humano en empresas de agroindustria de la guayaba en Colombia

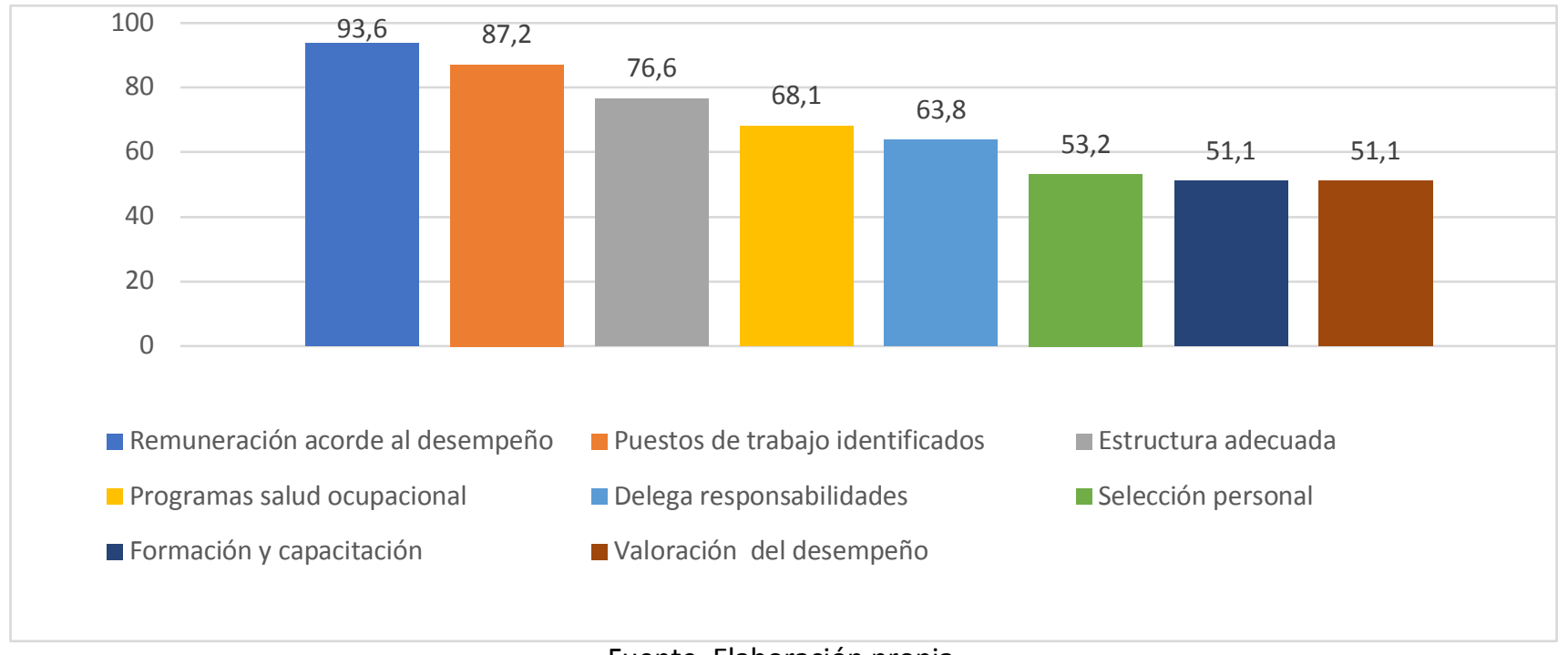

Fuente. Elaboración propia 


\subsection{Marketing operacional}

En este apartado como se dijo antes, se busca llevar a la práctica o ejecución lo propuesto, convirtiéndolo en acciones y decisiones tácticas y políticas comerciales; para el presente caso, se analizaron tres agrupaciones, la primera, se refiere a aspectos de producto y precio cuyos resultados (figura 6) dejan ver que en el $100 \%$ de las empresas está presente la estrategia basada en calidad del producto, en el 97,9\% cuentan con registro Invima, requisito obligatorio en productos comestibles y el restante porcentaje está en trámite; en el 93,6\% también consideran importante el servicio al cliente por su relación directa con la satisfacción del mismo, aspectos valorados de gran importancia por Serna (2014); Kotler y Lane (2016); Monferrer Tirado (2013), entre otros.

Figura 6

Cumplimiento en aspectos de producto y precio en empresas de agroindustria de la guayaba en Colombia

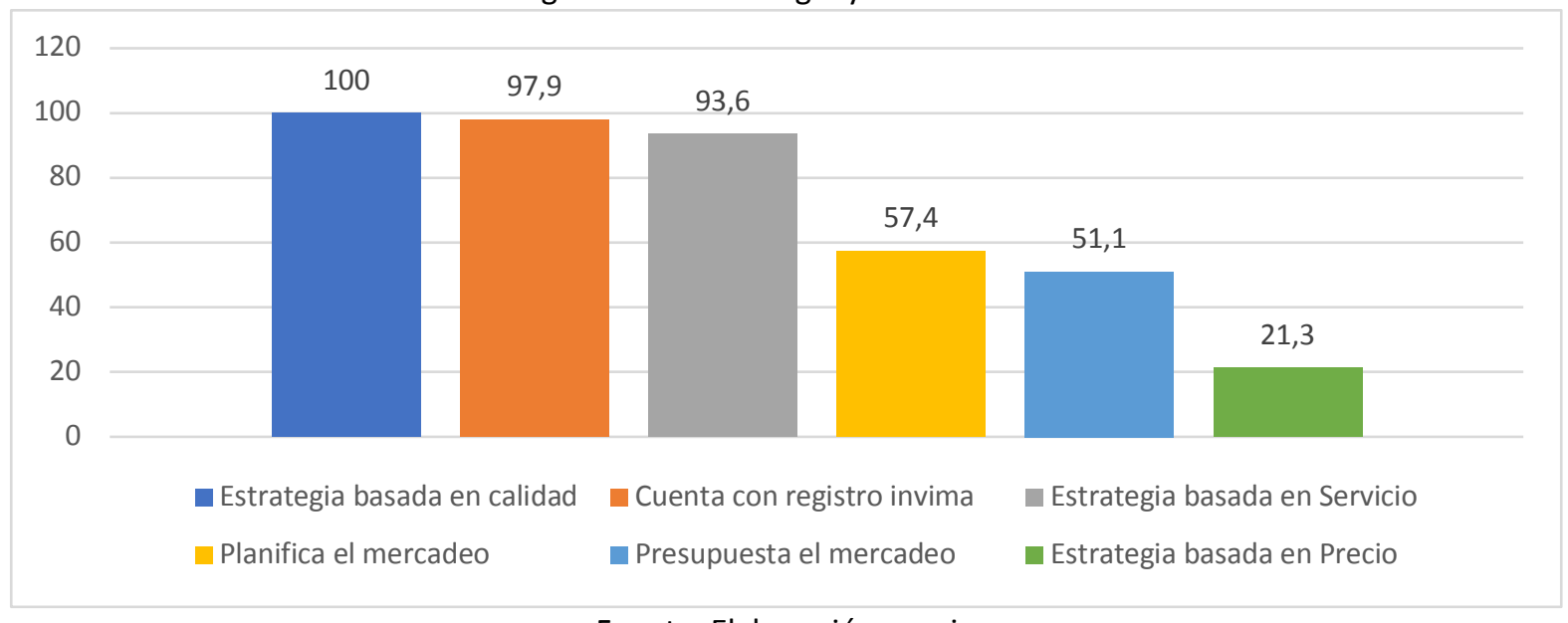

Fuente. Elaboración propia

En lo referente a planificar y asignar recursos para el mercadeo, se encontró que se cumple en el 57,4\% y 51,1\% respectivamente, porcentajes que ameritan mayor análisis para su incremento y aprovechamiento; de otra parte la estrategia basada en precio resultó ser no relevante para este sector empresarial, dado que se trata de productos de consumo popular que prácticamente están al alcance de todos los estratos, su resultado muestra un $21,3 \%$ pero no se puede considerar debilidad por las razones ya expuestas.

Otra agrupación analizada fue la correspondiente a aspectos propios de la distribución y comunicación sus resultados (tabla 5) dejan ver que los mayores porcentajes se ubican en ítems relacionados con el uso medios tradicionales de relacionamiento con el cliente (línea telefónica, correo electrónico, directorio empresarial) dejando en evidencia el rezago respecto al uso de las herramientas de Social Media.

\section{Tabla 5}

Cumplimiento en aspectos de distribución y comunicación en empresas de agroindustria de la guayaba en Colombia

\begin{tabular}{lcc}
\hline \multicolumn{1}{c}{ Aspectos valorados } & Frecuencia & Porcentaje \\
\hline Cuenta línea telefónica básica & 44 & 93,6 \\
Esta en por lo menos un directorio empresarial & 42 & 89,4 \\
Cuenta con canales de distribución adecuados & 40 & 85,1 \\
Cuenta con correo electrónico empresarial & 37 & 78,7 \\
Se comunica a través de internet con clientes y proveedores & 37 & 78,7 \\
Maneja Facebook & 23 & 48,9 \\
Realiza actividades de mercadeo a través de internet & 19 & 40,4 \\
Cuenta con página Web & 16 & 34 \\
Maneja Instagram & 6 & 12,8 \\
\hline
\end{tabular}

Fuente: Elaboración propia 
En lo referente a los aspectos analizados en evaluación y seguimiento, en el 91,5\% de las empresas, los objetivos y metas son verificables y comunicados al personal, de igual forma en ese mismo porcentaje se reconocen beneficios en la planificación; en cuanto a la calificación de las utilidades en el último año el 51,1\% las calificó como buenas, argumentado razones como buen movimiento del mercado, crecimiento de ventas, buenos ingresos, generación de ganancias respecto a la inversión y cumplimiento de las expectativas; mientras que el $46,8 \%$ las calificó como regulares por razones relacionadas con escasez de materia prima, competencia desleal y falta de incentivos de parte del gobierno.

Una vez realizado el análisis situacional se pudo establecer un resumen de las fortalezas, debilidades, oportunidades y amenazas en las empresas agroindustriales de la guayaba en Colombia como se muestra en la tabla 6, información de gran importancia para el planteamiento de objetivos y metas o replanteamiento de las existentes, al igual que el examen de las estrategias llevadas a cabo, en aras de mejorar los resultados del plan de marketing en estas empresas.

Tabla 6

Análisis FODA en empresas de agroindustria de la guayaba en Colombia

\begin{tabular}{|c|c|}
\hline Análisis Interno & Análisis externo \\
\hline Fortalezas & Oportunidades \\
\hline Direccionamiento estratégico & El consumidor globalizado \\
\hline Relación con el territorio & Recursos específicos y territoriales \\
\hline Saber-hacer tradicional & Inclusión del producto en el plan alimentario nacional \\
\hline Sus relaciones atrás y adelante & Acciones colectivas y coordinación con otros sectores \\
\hline Certificación de productos & económicos como el turismo \\
\hline Productos con anclaje territorial. & Articulaciones entre actores y empresas \\
\hline $\begin{array}{l}\text { Actores (individuales y colectivos) en procesos de } \\
\text { empoderamiento. } \\
\text { Denominación de Origen, Marca colectiva }\end{array}$ & $\begin{array}{l}\text { Consumidores social y ambientalmente } \\
\text { responsables, identificados con el desarrollo. } \\
\text { Para productos locales, procesos vinculados con el } \\
\text { territorio: Identificación Geográfica, }\end{array}$ \\
\hline Debilidades & Amenazas \\
\hline Presentación y promoción de los productos & Barreras no arancelarias y fitosanitarias \\
\hline Financiamiento & Competencia desleal con productos similares (sin \\
\hline Estrategias individuales & saber-hacer o anclaje territorial) \\
\hline Lejanía de los mercados importantes (nivel & Pérdida (o riesgo de) del saber-hacer. \\
\hline adquisitivo y especificidad de productos). & Falta de continuidad en los apoyos institucionales \\
\hline Planeación de corto plazo & Baja asociatividad y presencia de alianzas \\
\hline \multicolumn{2}{|l|}{ Tecnología } \\
\hline Baja utilización de internet para concretar ventas & \\
\hline
\end{tabular}

Fuente: Elaboración propia

\section{Conclusiones}

La agroindustria de la guayaba cuenta con una trayectoria de más de 200 años, el 100\% de las empresas analizadas lleva más de seis años de funcionamiento lo cual es un indicador de proyección, toda vez que la mayor mortalidad de empresas se da antes de cumplir los cinco años; también se destaca que el 51,1\% están dirigidas por mujeres y un alto porcentaje son empresas familiares.

El análisis del plan de marketing mostró que en la agrupación de direccionamiento estratégico el promedio de cumplimiento de los aspectos analizados fue del $86,4 \%$, en la de mercados del $72,9 \%$, relacionamiento con stakeholders $68,4 \%$, talento humano $68,1 \%$; en los aspectos referentes a producto y precio el $70,2 \%$, en comunicación y distribución $62,4 \%$ y en lo referente a evaluación y seguimiento $81,4 \%$; resultados que general 
se ubican por encima de $72,8 \%$ los cuales desde luego son susceptibles de mejora, donde en cada agrupación acorde al resultado del aspecto analizado se puede reformular o formular estrategias para un mejor desempeño empresarial y por ende del sector.

Finalmente, se pudo evidenciar que el hecho de contar con un plan de marketing contribuye a que las organizaciones puedan mejorar y potenciar los procesos comerciales, la comunicación e interacción con sus clientes y la búsqueda del cumplimiento de las necesidades y expectativas de los consumidores en lo referente a la calidad de los productos, mientras que se desarrollan actividades de control y análisis de información para la toma de decisiones respecto a temas operativos. Así mismo, desde la perspectiva estratégica, dicho plan permite establecer puntos de referencia para el crecimiento de las empresas agroindustriales, de esta manera los esfuerzos que se realicen en el futuro estarán en permanente interacción con el direccionamiento estratégico en busca del cumplimiento de los objetivos que se establezcan a mediano y largo plazo.

\section{Referencias bibliográficas}

Acevedo Navas, C., Cotes Escolar, J., Giraldo Oliveros, M., Juliao Esparragoza, D., Martínez Díaz, D., Ortiz Velásquez, M., Silva Guerra, H. (2016). Gerencia de Marketing. Barranquilla: ECOE.

Alonso, J; Ordoñez, P y Rivera, A (2017). La demanda de guayaba en Colombia. Corpoica. Ciencia y Tecnología Agropecuaria, 18(1): 25-45.

Artega, M., Ruiz, A., y Navas, W. (2017). Consideraciones sobre la realización de un plan de marketing para la industria de servicios. Dominio de las ciencias, 3(2), 894-907. doi: https://doi.org/10.23857/pocaip

Adel Vélez. (2015). Agroindustria del bocadillo veleño. Modelo de desarrollo económico local. Vélez: ProcasurSena. Recuperado de http://americalatina.procasur.org/images/2015/pdf_territo/Santander.pdf

Barrionuevo, C., Cárdenas, B., Cárdenas, H., Cabezas, R., y Sandoval, G. A. (2019). PESTEL analysis with neutrosophic cognitive maps to determine the factors that affect rural sustainability. Case Study of the South-Eastern plain of the province of Pinar del Río. Neutrosophic Sets and Systems (27), 217-227. Recuperado de https://digitalrepository.unm.edu/nss_journal/vol27/iss1/19/

Borges Andrade, J., Escobar, M., Palomino, J., Saldaña, R., y De Souza Silva, J. (1995). Planificación estratégica en la administración de la investigación agropecuaria. En Capacitación en planificación, seguimiento y evaluación para la administración. Brasil: Servicio Internacional para la Investigación Agrícola Nacional.

Estrada, K., Elidea, R., Cifuentes, L., y Ayovi, J. (2017). El plan de marketing y su importancia para el posicionamiento de las empresas. Polo del conocimiento, 2(5), 1187-1199

Ferrell, O. C., y Hartline, M. (2018). Estrategia de Marketing. México. Cengage.

García, Y., y Pérez, D. (2018). La importancia del Plan de Mercadotecnia en las Empresas Internacionales. Universidad autónoma de Nuevo León, Facultad de Contaduría y Administración. Recuperado de http://www.web.facpya.uanl.mx/vinculategica/Vincultagieca_4/34\%20GARCIA_PEREZ.pdf

Gobernación de Santander. (30 de 06 de 2017). Triunfo con sabor a bocadillo. Triunfo con sabor a bocadillo. Bucaramanga, Colombia. Recuperado de http://www.santander.gov.co/index.php/actualidad/item/569triunfo-con-sabor-a-bocadillo

González, M. (05 de 2015). Importancia macroeconómica de la agroindustria colombiana. Recuperado de agrounicauca.blogspot.com: http://agrounicauca.blogspot.com/2015/05/importancia-macroeconomicade-la.html\#: :text=Desde\%20sus\%20inicios\%20la\%20agroindustria,de\%20este\%20sector\%20y\%20su 
Jiménez, L. A., y Gamboa, R. (2016). El Endomarketing: aplicado al talento clave interno en el sector salud. Revista Mundo FESC, 8-19.

Kotler, P., y Lane, K. (2016). Dirección de Marketing. México. Pearson.

Lambin, J. J., Gallucci, C., y Sicurello, C. (2009). Dirección de marketing: gestión estratégica y operativa del mercado. México: McGraw-Hill.

Maldonado Pinto, J. E. (2013). Principios de marketing. Bogotá: Ediciones de la U.

Ministerio de Agricultura y Desarrollo Rural. (2015). Organización Cadena Agroalimentaria de la Guayaba y su Industria. Dirección de cadenas productivas. Bogotá: Minagricultura.

Ministerio de Agricultura y Desarrollo Rural. (2018). Cadena de guayaba indicadores e Instrumentos, septiembre 2018. Cadenas productivas, Bogotá.

Monferrer Tirado, D. (2013). Fundamentos de marketing. Castelló de la Plana: Publicaciones de la Universitat Jaume I.

Munera Alemán, J. L., y Rodríguez Escudero, A. I. (2020). Estrategias de marketing. Un enfoque basado en el proceso de dirección. Madrid: ESIC.

Muñiz González, R. (2015). Marketing en el siglo XXI. CEF. Recuperado de http://www. marketingxxi.com/Marketing-siglo-xxi.html

Sainz de Vicuña, J. (2018). El plan de marketing en la práctica. ESIC. Editorial.

Sánchez, A. (2015). Cómo realizar un buen Plan de Marketing y No Morir en el Intento. Kindle E-book.

Serna, H. (2014). Gerencia Estratégica. Bogotá: 3R editores.

Stanton, W., Etzel, M., y Walker, B. (2007). fundamentos de Marketing. México: McGraw Hill.

Superintendencia de Industria y Comercio (2017). Denominación de origen del bocadillo veleño. resolución N 35076 del 15 de junio de 2017. Recuperado de https://www.sic.gov.co/sites/default/files/documentos/092017/res-37562-bocadillo-velenio.pdf

Taipe, J. y Pazmiño, J. (2015). Consideración de los factores o fuerzas externas e internas a tomar en cuenta para el análisis situacional de una empresa. Revista Publicando, 2(2), 163-183.

Thompson, A., Strickland, A., Janes, A., Sutton, C., Peteraf, M., y Gamble, J. (2018). Administración Estratégica. teorías y Casos. México: McGraw Hill.

Toca Torres, C. E. (2009). Fundamentos del marketing: guía para su estudio y comprensión. Bogotá: Universidad del Rosario.

Villegas, J., y Escalante, J. E. (2016). Gerencia de Marca: un enfoque estratégico. Medellín: EAFIT.

Viteri, F., Herrera, L., y Bazurto, A. (2017). Las Tendencias del Marketing: Cuales son y definiciones. Revista Científica Mundo de la Investigación y el Conocimiento, 1(5), 974-988. doi:10.26820/recimundo/1.5.2017.974-988

Zárraga, L., Molina, V., y Corona, E. (2018). La satisfacción del cliente basada en la calidad del servicio a través de la eficiencia del personal y eficiencia del servicio. Un estudio empírico de la industria restaurantera. Revista de Estudios en Contaduría, Administración e Informática, año 7(18), 46 - 65. 
Zárraga, L., Molina, V., y Corona, E. (2013). Estudio de caso. Análisis de la aplicación de la segmentación de mercado como estrategia para las pequeñas empresas. Internacional administración \& finanzas, 6(5), 109119.

Zenea, M., Arcia, B., y Guerra, O. (2019). Metodología para el empleo de la herramienta del diagnóstico estratégico en Cooperativas no Agropecuarias. Estudios del desarrollo Social, 7(3), 192-206.

Esta obra está bajo una Licencia Creative Commons

Attribución-NoCommercial 4.0 International

(cc) BY-NC 